J. Austral. Math. Soc. 19 (Series A) (1975), 173-179.

\title{
ORTHOGONALITY RELATIONS ON ABELIAN GROUPS
}

\author{
G. DAVIS
}

(Received 18 January 1972; revised 17 July 1972)

Communicated by J. B. Miller

\begin{abstract}
An orthogonality relation is an abstract relation on a group having properties similar to the relation on a $l$-group given by $x \perp y$ if $|x| \wedge|y|=0$. A group $G$ with an orthogonality relation $\perp$ is isomorphically represented as a subgroup of the group $\Gamma$ of continuous global sections of a sheaf of groups. If the stalks of the sheaf are torsion-free and $G$ has and element 1 satisfying $1^{+}=(0)$ then $\Gamma$ can be ordered so that it is an $l$-group and $x \perp y$ if and only if $|x| \wedge|y|=0$ in $\Gamma$. An l-group $G$ is complemented if for all $x, y \in G$ there is an $a \in x^{+} \oplus x^{++}$with $y \in a^{++}$: equivalent conditions are given for $G$ to be complemented.
\end{abstract}

\section{Introduction}

A. I. Veksler (1967) introduced the concept of disjointness relation on a linear space, as an abstraction of the relation $\perp$ on vector lattices defined by $x \perp y$ if $|x| \wedge|y|=0$, and showed that for certain linear spaces $E$ with a disjointness relation $\perp$ it is possible to define a lattice-order on $E$ such that $x \perp y$ if and if and only if $|x| \wedge|y|=0$. In this paper the concept of disjointness, now called orthogonality, is extended to abelian groups. It is seen that every group $G$ with an orthogonality relation is (isomorphic with) a subgroup of a group $\Gamma$ of continous global sections of a sheaf of groups with a Boolean base space. When the group $G$ has an element 1 satisfying $1^{\perp}=(0)$ and the groups comprising the stalks in the sheaf representation are torsion-free then these stalks can be totally-ordered so that $\Gamma$ is a lattice-ordered group and $x \perp y$ if and only if $|x| \wedge|y|=0$.

Finally, verious characterizations are given of a class of $l$-groups that were called "weakly projectable" by Spirason and Strzelecki (to appear).

\section{Orthogonality relations}

Throughout, let $G$ be an abelian group. A relation $\perp$ on $G$ is a pre-orthogonality relation if 
(1) $x \perp y$ implies $y \perp x$

(2) $0 \perp x$ for all $x \in G$, where 0 is the identity of $G$

(3) $x \perp x$ implies $x=0$

(4) $x \perp a, y \perp a$ implies $x+y \perp a$.

For each non-empty subset $A$ of $G$ define $A^{\perp}=\{x \in G: x \perp a)$ for all $\left.a \in A\right\}$, $A^{\perp \perp}=\left(A^{\perp}\right)^{\perp}$. If $A=\{x\}$ is a singleton set then denote $A^{\perp}, A^{\perp \perp}$ by $x^{\perp}, x^{\perp \perp}$. An orthogonality relation on $G$ is a pre-orthogonality relation $\perp$ for which $x \perp y$ is equivalent to $x^{\perp \perp} \cap y^{\perp \perp}=(0)$. Using Frink's axioms (1941) for a Boolean algebra, it is readily seen that when $\perp$ is an orthogonality relation the class $B(G)$ of subsets of $G$ of the form $A^{\perp}$, ordered by inclusion, is a complete Boolean algebra with $\bigcap_{\alpha} A_{\alpha}^{\perp}=(\cup A \alpha)^{\perp}$ and $A^{\perp \perp}$ as the complement of $A^{\perp}$.

The Stone space of $B(G)$ (i.e., the set of prime ideals of $B(G)$ equipped with the hull-kernel topology) will be denoted by $Q$. For each $t \in Q$ a subset $G_{t}$ of $G$ is defined by $G_{t}=\left\{x \in G: x^{\perp \perp} \in t\right\}$. It is readily seen that each $G_{t}$ is a subgroup of $G$ and $\cap_{t \in Q} G_{t}=(0)$. Using the method of Dauns and Hoffman (1966) it can be seen that there is a sheaf of groups with base space $Q$ and stalks $G / G_{t}, t \in Q$, and an isomorphism a given by $\hat{x}(t)=x+G_{t}$ mapping $G$ into the group $\Gamma$ of continuous global sections of this sheaf, such that $x \perp y$ if and only if for each $t \in Q$ either $\hat{x}(t)=0$ or $\hat{y}(t)=0$. This sheaf representation is more special than the obvious representation of $G$ as a sub-direct product of the groups $G / G_{t}$ only in that $Q$ has a topology and the disjoint union of the groups $G / G_{t}$ is given the finest topology for which each $\hat{x}$ is continuous. $\Gamma$ then consists of all choice functions $Q \rightarrow \cup G / G_{t}$ that agree locally with some $\hat{x}$. Since $Q$ is compact Hausdorff and has a base for the open sets consisting of the closed open sets $Q_{A_{1}^{\perp}}=\left\{t \in Q: A^{\perp} \notin t\right\}, A \subseteq G$, then for any $\sigma \in \Gamma$ there is a finite closed-open partition $Q_{A_{i}}^{+}, \cdots, Q_{A_{n}^{+}}^{+}$of $Q$, and $x_{1}, \cdots, x_{n} \in G$ such that $\sigma=\Sigma I\left(Q_{A_{i}^{*}} ; x_{i}\right)$ where

$$
I\left(Q_{A_{i}} ; x_{i}\right)(t)=\left\{\begin{array}{ll}
\hat{x}(t) & \text { if } t \in Q_{A_{i}} \\
0 & \text { if } t \in Q_{A_{i}}
\end{array} .\right.
$$

A necessary and sufficient condition for $\hat{G}=\{\hat{x}: x \in G\}$ and $\Gamma$ to be isomorphic is $G=A^{\perp} \oplus A^{\perp \perp}$ for all $A^{\perp} \in B(G)$.

\section{Lattice-ordering $\Gamma$}

It will be assumed in this section that $\perp$ is an orthogonality relation on the abelian group $G$.

LEMma 2.1. The quotient groups $G / G_{t}$ are all torsion-free if and only if $x^{\perp}=(m x)^{\perp}$ for all $x$ in $G$ and all integers $m>1$.

Proof. Assume $x^{\perp}=(m x)^{\perp}$ for all $x$ in $G$ and all $m \geqq 1$. I $m x \in G_{t}$ then $x^{\perp \perp}=(m x)^{\perp \perp} \in t$ so $x \in G_{t}$ and $G / G_{t}$ is torsion free. If each $G / G_{t}$ is torsion-free and $y \perp m x$ then $y \notin G_{t}$ implies $m x \in G_{t}$ so $x \in G_{t}$ and thus $y \perp x$. 
Proposinon 2.2. Let $G$ have an orthogonality relation $\perp$ satisfying $x^{\perp}=(m x)^{\perp}$ for all $x \in G, m \geqq 1$. For each $t \in Q$ let $P_{t}$ be a positive set defining a total order on $G / G_{t}$ compatible with the group structure. Define a partial order on $\Gamma$ by $\sigma \geqq 0$ if $\sigma(t) \in Q$. Then $\Gamma$ is lattice-ordered if for each $x \in G$ the set $\operatorname{Pos}(x)=\{t \in Q: \hat{x}(t)>0\}$ is open in $Q$.

Proof. Each $G / G_{t}$ is abelian and torsion-free by lemma 2.1 and therefore admits a total order $P_{t}$. Suppose that $\operatorname{Pos}(x)$ is open for each $x \in G$. Then the set $\{t \in Q: \hat{x}(t) \geqq 0\}=Q \mid \operatorname{Pos}(-x)$ is closed and contains $\operatorname{Pos}(x)$ yet if $\hat{x}\left(t_{0}\right)=0$ then there is a basic closed-open neighbourhood $Q_{A}{ }^{+}=\left\{t \in Q: A^{\perp} \notin t\right\}$ of $t_{0}$ such that $\hat{x}(t)=0$ for all $t \in Q_{A}{ }^{\text {}}$ so that $Q_{A}{ }^{+} \cap \operatorname{Pos}(x)$ is void. Hence $\operatorname{Pos}(x)$ is closed and therefore closed-open. The function $\hat{x} \vee 0$ mapping $Q$ into $\bigcup_{t \in Q} G / G_{t}$ ) according to $t \leftrightarrow \max \{\hat{x}(t), 0\}$ is the supremum of $\hat{x}$ and 0 in the ordered group of all (not necessarily continuous) global sections. However, $\hat{x} \vee 0=I(\operatorname{Pos}(x) ; x$ where

$$
I(\operatorname{Pos}(x) ; x)(t)= \begin{cases}\hat{x}(t) & \text { if } t \in \operatorname{Pos}(x) \\ 0 & \text { if } t \in \operatorname{Pos}(x)\end{cases}
$$

so $\hat{x} \vee 0 \in \Gamma$. For $\sigma=\sum_{i} I\left(Q_{A_{i}^{*}}: x_{i}\right) \in \Gamma$, where $\left\{Q_{A_{i}^{2}}\right\}$ is a finite closed-open partition of $Q$,

$$
\begin{aligned}
\sigma \vee 0(t) & =\max \{\sigma(t), 0\} \\
& =\sum_{i} I\left(Q_{A_{i}^{2}} \cap \operatorname{Pos}\left(x_{i}\right) ; x_{i}\right)
\end{aligned}
$$

is in $\Gamma$, so that $\Gamma$ is lattice-ordered.

THEOREM: 2.3. If $G$ has an orthogonality relation $\perp$ satisfying $x^{\perp}=(m x)^{\perp}$ for all $x$ in $G, m \geqq 1$ and an element 1 satisfying $1^{\perp}=(0)$ then the group $\Gamma$ can be lattice-ordered so that $\sigma \perp \tau$ if and only if $|\sigma| \wedge|\tau|=0$ for $\sigma, \tau$ in $\Gamma$.

Proof. Since $1^{\perp}=(0)$ then $1 \notin G_{t}$ for all $t \in Q$. For each $t \in Q$ there is a positive set in $G / G_{t}$ such that for each $x \in G$ the set $\operatorname{Pos}(x)=\{t \in Q: \hat{x}(t)>0$ in $\left.G / G_{t}\right\}$ is open. Consider the positive sets $\left\{m 1+G_{t}: m=0,1,2, \cdots\right\}:$ for $x \in G$, $\operatorname{Pos}(x)=\bigcup_{m=0}^{\infty}\{t \in Q: \overparen{x-n} 1(t)=0\}$ which, as a union of open sets, is open. The class $K$ of strings $\left(P_{t}\right)$ such that each $P_{t}$ is a positive set in $G / G_{t}$ containing $1+G_{t}$ and such that $\operatorname{Pos}(x)=\left\{t \in Q: 0 \neq x+G_{t} \in P_{t}\right\}$ is open for each $x \in G$ is inductive when ordered by $\left(P_{t}\right)_{t \in Q} \geqq\left(P_{t}^{\prime}\right)_{t \in Q}$ if $P_{t} \supseteq P_{t}^{\prime}$ for all $t \in Q$, so that maximal elements of $K$ follow from the axiom of choice. Let $\left(P_{t}\right)_{t \in Q}$ be maximal in $K$. For $x \in G$ the closure $\overline{\operatorname{Pos}(x)}$ of $\operatorname{Pos}(x)$ is closed-open in $Q$. If $t \in Q$ define $P_{t}^{\prime}=\left\{x+G_{t}: t \in \overline{\operatorname{Pos}(x)}\right\}$. If $x+G_{t} \in P_{t}^{\prime} \cap\left(-P_{t}^{\prime}\right)$ then $t \in \overline{\operatorname{Pos}(x)} \cap \overline{\operatorname{Pos}(-x)}$ which, however, is void since $\overline{\operatorname{Pos}(x)}, \overline{\operatorname{Pos}(-x)}$ are disjoint open sets with open closures. For each $t \in Q$ the set $P_{t}^{\prime} \cup\{0\}$ is therefore a positive set in $G / G_{t}$ containing $P_{t}$. Furthermore, for $x \in G$, 


$$
\begin{aligned}
\operatorname{Pos}^{\prime}(x) & =\left\{t \in Q: x+G_{t} \in P_{t}^{\prime}\right\} \\
& =\{t \in Q: t \in \overline{\operatorname{Pos}(x)}\}=\overline{\operatorname{Pos}(x)} \text { is closed-open. }
\end{aligned}
$$

The maximality of $\left(P_{t}\right)_{t \in Q}$ in $K$ therefore gives that $\operatorname{Pos}(x)$ is closed-open for each $x \in G$. Now suppose that for some $t_{0} \in Q, P_{t_{0}}$ does not define an isolated order on $G / G_{t_{0}}$, so there is an $a+G_{t_{0}} \in P_{t_{0}}$ and an integer $m_{0} \geqq 2$ such that $m_{0} a+G_{t_{0}} \in P_{t_{0}}$.

Then, $t_{0} \in \operatorname{Pos}\left(m_{0} a\right)$ so there is an open neighbourhood $Q_{A}+$ of $t_{0}$ contained in $\operatorname{Pos}\left(m_{0} a\right)$. Also, $t_{0} \notin \operatorname{Pos}(a)$ so there is an open neighbourhood $Q_{B}{ }^{+}$of $t_{0}$ contained in $Q \backslash \operatorname{Pos}(a)$. Then for $t \in Q_{A}{ }^{+} \cap Q_{B}{ }^{+}, P_{t}$ does not define an isolated order on $G / G_{t}$. Consider the string $\left(P_{t}^{\prime}\right)_{t \in Q}$ with

$$
P_{t}^{\prime}=\left\{\begin{array}{l}
P_{t} \text { if } t \in Q_{A}{ }^{+} \cap Q_{B}{ }^{2}, \\
\left\{m a+y+G_{t}: m=0,1,2, \cdots, y+G_{t} \in P_{t}\right\} \\
\text { if } t \in Q_{A}{ }^{+} \cap Q_{B}{ }^{+}
\end{array}\right.
$$

Each $P_{t}^{\prime}$ is a semigroup properly containing $P_{t}$. If $P_{t}^{\prime}$ is not a positive set for some $t \in Q_{A} \cap \cap Q_{B}$ then $n_{1} a+y_{1}+G_{t}=-n_{2} a-y_{2}+G_{t}$ for some integers $n_{1}$, $n_{2} \geqq 1$, and $y_{1}+G_{t}, y_{2}+G_{t} \in P_{t}$. Then $\left(n_{1}+n_{2}\right) a+G_{t}=-\left(y_{1}+y_{2}\right)+G_{t} \epsilon$ $-P_{t}$ and $m_{0}\left(n_{1}+n_{2}\right) a+G_{t} \in P_{t}$ (since $m_{0} a(t)>0$ for $t \in Q_{A}{ }^{+} \cap Q_{B}{ }^{2}$ ) so that $m_{0}\left(n_{1}+n_{2}\right) a+G_{t}=0$ which means $a \in G_{t}$ since $G / G_{t}$ is torsion-free. This contradicts the choice of $a \in G$ so that each $P_{t}^{\prime}$ is a positive set in $G / G_{t}$. For $x \in G$ put $\operatorname{Pos}^{\prime}(x)=\left\{t \in Q: 0 \neq x+G_{t} \in P_{t}^{\prime}\right\}$. Suppose $t_{1} \in \operatorname{Pos}^{\prime}(x)$. If $t_{1} \in Q_{A}{ }^{+} \cap Q_{B}{ }^{+}$ then $t_{1} \in \operatorname{Pos}(x)$ so there is an open neighbourhood of $t_{1}$ contained in $\operatorname{Pos}(x)$ and hence in $\operatorname{Pos}^{\prime}(x)$. If $t_{1} \in Q_{A}{ }^{+} \cap Q_{B}$ then $\hat{x}\left(t_{1}\right)=\widetilde{m a+y} y\left(t_{1}\right) \neq 0$, so there is an open neighbourhood $Q_{c}{ }^{+}$of $t_{1}$ such that $\hat{x}(t)=\overparen{m a+y} y(t) \neq 0$ for $t \in Q_{c}^{+}$. Also, there is an open neighbourhood $Q_{D}{ }^{+}$of $t_{1}$ such that $\hat{y}(t)>0$ for $t \in Q_{D}{ }^{+}$. Thus $Q_{C}+\cap Q_{D^{+}} \supseteq \operatorname{Pos}^{\prime}(x)$. The set $\operatorname{Pos}^{\prime}(x)$ is therefore open for each $x \in G$. This contradicts the maximality of $\left(P_{t}\right)_{t \in Q}$ in $K$ so that each $P_{t}$ defines an isolated order on $G / G_{t}$. Now suppose that for some $t \in Q, P_{t}$ does not define a total order on $G / G_{t}$, so that for some $a \in G, a+G_{t} \in P_{t} \cup\left(-P_{t}\right)$. That is $t \in \operatorname{Pos}(x) \cap \operatorname{Pos}(-x)$ which is an open set, so for $t_{1}$ in some open neighbourhood $Q_{A}{ }^{+}$of $t, P_{t}$ does not define a total order on $G / G_{t_{1}}$. As before, consider the string $\left(P_{t}^{\prime}\right)_{t \in Q}$ with

$$
P_{t}^{\prime}=\left\{\begin{aligned}
P_{t} \text { if } t \in Q_{A^{+}}, & \\
\left\{m a+y+G_{t}:\right. & \left.m=0,1,2, \cdots, y+G_{t} \in P_{t}\right\} \\
& \text { if } t \in Q_{A}^{+}
\end{aligned}\right.
$$

Each $P_{t}^{\prime}$ is a semigroup containing $P_{t}$ in $G / G_{t}$. If $P_{t}^{\prime}$ is not a positive set for some $t \in Q_{A}{ }^{+}$then, as before, $m a+G_{t} \in-P_{t}$ for some $m \geqq 1$. Since $P_{t}$ defines an 
isolated order on $G / G_{t}$ then $a+G_{t} \in-P_{t}$, contrary to assumption. The maximality of $\left(P_{t}\right)_{t \in Q}$ therefore gives that each $P_{t}$ defines a total order on $G / G_{t}$. The group $\Gamma$ is therefore lattice-ordered by $\sigma \geqq 0$ if $\sigma(t) \in P_{t}$ for all $t \in Q$. Since $|\sigma|(t)$ $=\max \{\sigma(t),-\sigma(t)\}$ in $G / G_{t}$ and $\sigma \wedge \tau(t)=\min \{\sigma(t), \tau(t)\}$ in $G / G_{t}$, then $|\sigma| \wedge|\tau|=0$ if only if for each $t \in Q$ either $\sigma(t)=0$ or $\tau(t)=0$.

\section{Lattice-groups}

In this section $G$ will denote an abelian lattice-group. The relation defined on $G$ by $x \perp y$ if $|x| \wedge|y|=0$ is an orthogonality relation with

$$
\begin{aligned}
& x^{\perp \perp} \cap v^{\perp \perp}=(|x| \wedge|y|)^{\perp \perp} \\
& x^{\perp \perp} \vee y^{\perp \perp}=(|x| \vee|y|)^{\perp \perp},
\end{aligned}
$$

and the subgroups $G_{t}$ are prime lattice-ideals. The results $3.1,3.3$ are due to Spirason and Strzelecki (to appear).

Proposition 3.1. A subgroup $I \subseteq G$ is of the form $G_{t}$ for some $t \in Q$ if and only if

$$
\begin{aligned}
& \text { (1) } x \perp y \text { implies } x \in I \text { or } y \in I \\
& \text { (2) } x \in I \text { implies } x^{\perp \perp} \subseteq I \\
& \text { (3) } x \in I \text { implies } x^{\perp} \neq(0)
\end{aligned}
$$

An immediate corollary to this is that every minimal prime lattice-ideal of $G$ is of the form $G_{t}$, for some $t \in Q$. Recall that a prime lattice-ideal $M$ is minimal prime if $M$ is minimal in the class of prime lattice-ideals of $G$ : a necessary and sufficient condition for a prime lattice-ideal $M$ to be minimal prime is that for each $x \in M$ there is a $y \notin M$ with $x \perp y$.

Definition 3.2. The lattice-group $G$ is said to be complemented if for all $x, y \in G$ there is an $a \in x^{\perp \perp} \oplus x^{\perp}$ such that $y \in a^{\perp-!}$.

THEOREM 3.3. $G$ is complemented if and only if every subgroup $G_{t} \neq G$ is a minimal prime lattice-ideal.

The class of minimal prime lattice ideals of $G$ will be denoted by $M(G)$. For $x \in G$ the class $M(x) \subseteq M(G)$ is defined by $M(x)=\{M \in M(G): x \notin M\}$. The set $\mu_{G}=\{M(x): x \in G\}$ forms a closed-open base for the open sets for a Hausdorff topology on $M(G)$. In fact, for $x, y \in G$,

$$
M(x) \cap M(y)=M(|x| \wedge|y|)
$$

and

$$
M(x) \cup M(y)=M(|x| \vee|y|)
$$


The class of subgroups $G_{t} \neq G$ is denoted by $V(G)$. For $x \in G$, the class $V(x)$ is defined by $V(x)=\left\{G_{t} \neq G: x \notin G_{t}\right\}$. For $x, y \in G$,

$$
V(x) \cap V(y)=V(|x| \wedge|y|)
$$

and

$$
V(x) \cup V(y)=V(|x| \vee|y|) .
$$

The set $v_{G}=\{V(x): x \in G\}$ then forms a compact-open base for the open sets for a topology on $V(G)$, which is compact if and only if $G$ has an element 1 satisfying $1^{\perp}=(0)$. Further it is readily seen that for $x, y \in G, V(x)=V(y)$ if and only if $x^{\perp}=y^{\perp}$.

THEOREM 3.4. The following are equivalent:

(1) $G$ is complemented

(2) $V(G)=M(G)$

(3) $v_{G}$ is relatively complemented

(4) $V(G)$ is a Hausdorff space

(5) Each $V(x) \in v_{G}$ is closed in $V(G)$.

Proof. If every $G_{t} \neq G$ is minimal prime and $G_{t_{1}} \neq G_{t_{2}} \notin V(G)$ then there is an $x \in G_{t_{1}}$ such that $x \notin G_{t_{2}}$, and a $y \in G_{t_{1}}$ such that $x \perp y$. Then $G_{t_{1}} \in V(y)$, $G_{t_{2}} \in V(x)$ and $V(y) \cap V(x)$ is void. Conversely, if $V(G) \neq M(G)$ then there is $a G_{t} \neq G$ that is not minimal prime. Then $G_{t}$ contains a minimal prime $G_{t_{1}}$, and $G_{t}, G_{t}$ cannot be Hausdorff-separated. Thus (2) is equivalent to (4). Suppose that $G$ is complemented. Take $V(x) \in v_{G}$ and $V(y) \subseteq V(x)$ so that $y^{\perp \perp} \subseteq x^{\perp \perp}$. Then there exist $a \in y^{\perp}, b \in y^{\perp \perp}$ such that $x^{\perp \perp} \subseteq(a+b)^{\perp \perp}=a^{\perp \perp} \vee b^{\perp \perp}$. Then

$$
\begin{gathered}
(|x| \wedge|a|)^{\perp \perp} \subseteq x^{\perp \perp} \text { and }(|x| \wedge|a|)^{\perp \perp} \vee y^{\perp \perp}=\left(x^{\perp \perp} \wedge a^{\perp \perp}\right) \vee y^{\perp \perp} \\
=\left(x^{\perp \perp} \vee y^{\perp \perp}\right) \wedge\left(a^{\perp \perp} \vee y^{\perp \perp}\right)=x^{\perp \perp} .
\end{gathered}
$$

Also, $|x| \wedge|a| \perp y$ so that $(|x| \wedge|a|) \cap V(y)$ is void, and $V(|x| \wedge|a|)$ $\cup V(y)=V(x)$. That is $v_{G}$ is relatively complemented. On the other hand if $v_{G}$ is relatively complemented and $x, y \in G$ then $V(x) \subseteq V(|x| \wedge|y|)$ so there is an $x^{\prime} \in G$ such that $x^{\prime} \perp x$ and $\left(x^{\prime}\right)^{\perp \perp} \vee x^{\perp \perp}=x^{\perp \perp} \wedge y^{\perp \perp}$. Then $y \in\left(x^{\prime}\right)^{\perp \perp} \vee x^{\perp \perp}=\left(x^{\prime}+x\right)^{\perp \perp}$ so that $G$ is complemented. That is (1) is equivalent to (3).

Suppose that each $V(x)$ in $v_{G}$ is closed and $G_{t} \neq G$. If $x \in G_{t}$ then $t \in V(x)$ so there is a basic open set $V(y)$ with $t \in V(y) \subseteq V(G) \backslash V(x)$. That is $y \perp x$ and $y \notin G_{t}$, so that $G_{t}$ is minimal prime. If each $G_{t} \neq G$ is minimal prime then $V(G)=M(G)$ so that each $V(x)=M(x)$ is closed. 


\section{References}

J. Dauns and K. H. Hofmann (1966), 'The representation of biregular rings by sheaves', Math. $Z$. 91, 103-123.

G. T. Spira son and E. Strezelcki, 'A note on Pi-ideals' (to appear).

A.I. Veksler (1967), 'Linear spaces with disjoint elements and their conversion into vector lattices', LLeningrad Gos. Ped. Inst. Ucen. Zap. 328, 19-43.

O. Frink (1941), 'Representations of Boolean algebras', Bull. Amer. Math. Soc. 47, 755-756.

La Trobe University

Bundoora 3083 Australia 